\title{
Reduced EBP50 expression levels are correlated with unfavorable clinicopathological features of extrahepatic bile duct carcinoma and promote the proliferation and migration of QBC939 cells
}

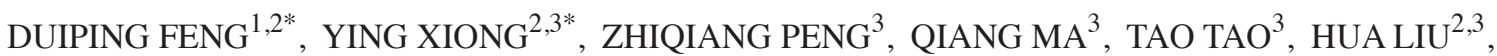 \\ JIANFANG LIANG ${ }^{4}$, ZHIGANG WEI ${ }^{5}$, JUNFANG ZHENG ${ }^{2,3}$, LEI WANG ${ }^{6}$ and HUI ZHANG ${ }^{1}$ \\ ${ }^{1}$ Department of Radiology, The First Hospital of Shanxi Medical University, Taiyuan, Shanxi 030001; \\ ${ }^{2}$ Beijing Key Laboratory for Tumor Invasion and Metastasis, Cancer Institute of Capital Medical University; \\ ${ }^{3}$ Department of Biochemistry and Molecular Biology, Capital Medical University, Beijing 100069; \\ Departments of ${ }^{4}$ Pathology and ${ }^{5}$ General Surgery, The First Hospital of Shanxi Medical University, Taiyuan, Shanxi 030001; \\ ${ }^{6}$ Department of Urology, Beijing Friendship Hospital, Capital Medical University, Beijing 100050, P.R. China
}

Received February 6, 2016; Accepted October 27, 2016

DOI: $10.3892 / 01.2017 .5789$

\begin{abstract}
The present study aimed to clarify the association between ezrin-radixin-moesin-binding phosphoprotein-50 (EBP50) expression level and the tumor phenotype and clinicopathological features of extrahepatic bile duct carcinoma. Tissue samples from patients with extrahepatic bile duct carcinoma (54 cases) and patients with normal bile duct epithelia from gallbladder of cholecystitis (20 cases) were collected, and immunohistochemical staining was used to detect the expression levels of EBP50 in these tissues. In addition, small interfering (si)RNA-EBP50 was used to knock down the expression of EBP50 in the QBC939 human cholangiocarcinoma (CC) cell line. The effect of EBP50 expression on QBC939 cell proliferation and migration was analyzed using the Cell Counting kit- 8 and wound healing assays, respectively. EBP50 expression was significantly downregulated in CC tissue samples $(\mathrm{P}<0.01)$, with low EBP50 expression levels positively correlated with a high pathological stage and a poor differentiation degree $(\mathrm{P}<0.01$ and $\mathrm{P}<0.001$, respectively). EBP50 expression in QBC939 cells was knocked down by $\leq 80 \%$ using siRNA-EBP50, and EBP50
\end{abstract}

Correspondence to: Dr Hui Zhang, Department of Radiology, The First Hospital of Shanxi Medical University, 85 Jiefang South Road, Taiyuan, Shanxi 030001, P.R. China

E-mail: zhanghui_mr@163.com

Dr Lei Wang, Department of Urology, Beijing Friendship Hospital, Capital Medical University, 95 Yong An Road, Xi Cheng, Beijing 100050, P.R. China

E-mail: 1049480155@qq.com

*Contributed equally

Key words: ezrin-radixin-moesin-binding phosphoprotein-50, cholangiocarcinoma, pathological stage, migration knockdown significantly promoted QBC939 cell proliferation, as compared with the vector control cells $(\mathrm{P}=0.04)$. EBP50 knockdown also significantly enhanced the wound healing ability of $\mathrm{QBC} 939$ cells $(\mathrm{P}=0.02)$. These results demonstrated that EBP50 expression levels are significantly correlated with a malignant phenotype in patients with $\mathrm{CC}$, and decreased expression levels of EBP50 may promote CC cell proliferation and migration. These findings provide insight into novel potential diagnostic and therapeutic approaches for patients with CC.

\section{Introduction}

Cholangiocarcinoma (CC) is a primary malignancy of the epithelial lining of the biliary tree with a poor prognosis (1). The 5-year survival rates are 15-35\% for resectable CC and $<5 \%$ for unresectable CC (2-6). The global incidence of this disease is steadily rising (7-9). Extrahepatic bile duct carcinoma accounts for 20-25\% of CC cases (10), and surgical resection remains the main curative option (11). Additionally, the impact of systemic chemotherapy on the survival of patients with CC is minimal $(2,3)$. To develop effective and targeted therapies, an improved understanding of the molecular mechanisms underlying the development and progression of this disease is required.

Ezrin-radixin-moesin-binding phosphoprotein-50 (EBP50) has been reported to have a role in liver and biliary-associated cancer, and is able to promote the development of liver cancer (12). EBP50 may also be a biomarker of advanced-stage malignancies in hepatocellular carcinoma (13). However, Peng et al (14) demonstrated that EBP50 executed a tumor-suppressor function via the $\beta$-catenin/E-cadherin signaling pathway in human hepatocellular cancer. Similarly, in biliary cancer cells, it has been observed that the downregulation of EBP50 stimulates epidermal growth factor receptor (EGFR) activity and is able to induce features of an epithelial-mesenchymal transition (EMT) phenotype (15), suggesting a tumor-suppressing role for EBP50. Therefore, the 
role of EBP50 in liver and biliary-associated cancer has yet to be elucidated.

In the present study, it was observed that the EBP50 expression levels were downregulated and correlated with disease stage and degree of differentiation in extrahepatic bile duct carcinoma tissue. Furthermore, EBP50 knockdown significantly enhanced cell proliferation and migration abilities, suggesting a tumor-suppressor function for EBP50. These results may aid the understanding of the pathogenesis of $\mathrm{CC}$ and provide a novel target for treatment.

\section{Materials and methods}

Patient tissues. The present retrospective study was approved by the Medical Ethics Committee of The First Hospital of Shanxi Medical University (Taiyuan, China), and was conducted according to the approved guidelines. Informed consent regarding the use of clinical tissue specimens was received from each patient. Surgical tissue specimens were obtained by surgical resection between January 2005 and December 2007 at The First Hospital of Shanxi Medical University, including 54 extrahepatic bile duct carcinoma cases (Hilar cholangiocarcinoma and gallbladder carcinoma; age range, 5-86; 29 males and 25 females), as well as normal bile duct epithelia from the gallbladder of 20 cholecystitis cases (Table I). All specimens obtained from the patients were histologically confirmed by a pathologist. Tumor stages were histologically classified according to the 2010 American Joint Cancer Committee tumor-node-metastasis (TNM) classification (stages I-IV) (16). Tumor differentiation degrees were defined according to the World Health Organization criteria (well differentiated, moderately differentiated and poorly differentiated).

Immunohistochemistry (IHC) assay. Formalin-fixed paraffin-embedded tissue samples were cut into 4 - $\mu \mathrm{m}$-thick sections, mounted on slides and dried at $70^{\circ} \mathrm{C}$ for $45 \mathrm{~min}$. Following deparaffinization in xylene and rehydration through decreasing graded alcohol and PBS washes ( $\mathrm{pH} 7.5)$, endogenous peroxidase activity was blocked by incubation with absolute methanol and 3\% hydrogen peroxide for $10 \mathrm{~min}$. Antigen retrieval was performed by sterilizing sections in $0.01 \mathrm{~mol} / 1$ citrate buffer $(\mathrm{pH} 6.0)$ at $98^{\circ} \mathrm{C}$ for $20 \mathrm{~min}$. Tissue sections were incubated at the $4^{\circ} \mathrm{C}$ overnight with an anti-EBP50 antibody (dilution, 1:100; cat. no., ab109430; Abcam, Cambridge, MA, USA). Subsequently, tissue sections were incubated with goat anti-rat immunoglobulin G H\&L (phycoerythrin) secondary antibody (dilution, 1:200; cat. no., ab7010; Abcam) at room temperature for $1 \mathrm{~h}$. The immunoreaction was visualized using a biotin-free dextran chain-based EnVision detection system (Dako, Glostrup, Denmark) using 3,3'-diaminobenzidine (Dako) as a chromogen. The tissue sections were counterstained with hematoxylin. Negative controls were created by omitting the primary antibodies, whereas QBC939 cell blocks (American Type Culture Collection, Manassas, VA, USA) were used as positive controls.

Cell culture and small interfering (si)RNA-mediated transient EBP50 knockdown. QBC939 cells were cultured in Dulbecco's modified Eagle's medium $\left(\right.$ Gibco $^{\mathrm{TM}}$; Thermo Fisher Scientific, Inc., Waltham, MA, USA) supplemented with $10 \%$ fetal bovine serum (HyClone; GE Healthcare Life Sciences, Logan, UT, USA) and $1 \%$ antibiotic-antimycotic agent, including amphotericin B, streptomycin and penicillin $\left(\mathrm{Gibco}^{\mathrm{TM}}\right.$; Thermo Fisher Scientific, Inc.). siRNA duplexes directed against EBP50 (5'-GUCGACCACCAGCAGGCGCACGGC GUUG-3') and control scrambled RNA interference (RNAi; 5'-UCCAGACGGCGCAGUGGGCGACCGCUAC-3') were synthesized by Sigma-Aldrich (Merck Millipore, Darmstadt, Germany). QBC939 cells were grown to $80 \%$ confluence in 90-mm dishes, transfected with $10 \mu \mathrm{l}$ Lipofectamine ${ }^{\circledR} 2000$ (Invitrogen; Thermo Fisher Scientific, Inc.) according to the manufacturer's protocol, and mixed with 180 pmol synthetic EBP50 siRNA. The transfected QBC939 cells were cultured for $48 \mathrm{~h}$ at $37^{\circ} \mathrm{C}$ with $5 \%$ carbon dioxide. The cells were used $48 \mathrm{~h}$ post-transfection.

Western blot analysis. The cells were collected and lysed in radioimmunoprecipitation assay buffer (Beijing Kawin Bio-Tech Co., Ltd., Beijing, China) in the presence of protease inhibitors (\#ab201119; Abcam) for $20 \mathrm{~min}$, in order to extract the total protein from cells transfected with EBP50 siRNA (siEBP50) or scrambled RNAi negative control (siRNA-NC). Protein levels were quantified by bicinchoninic acid assay (Beijing Kawin Biotech Co., Ltd.). Protein samples were separated by $8 \%$ SDS-PAGE and transferred to polyvinylidene fluoride membranes. The membranes were blocked in a blocking buffer [5\% non-fat dry milk in TBS with Tween-20 (TBST) buffer] for $1 \mathrm{~h}$ at room temperature, and then incubated with primary antibodies in blocking buffer overnight at $4^{\circ} \mathrm{C}$. The blots were washed three times with TBST buffer and incubated with an antibody targeting EBP50 (\#611160; BD Biosciences, San Jose, CA, USA; dilution, 1:1,000) or $\beta$-actin (\#3700; Cell Signaling Technology, Inc., Danvers, MA, USA; dilution, $1: 1,000)$ overnight at $4^{\circ} \mathrm{C}$, followed by incubation with secondary antibodies conjugated with horseradish peroxidase (\#RPN4201; GE Healthcare Bio-Sciences, Pittsburgh, PA, USA; dilution, 1:3,000) for $1 \mathrm{~h}$ at room temperature. Finally, the blots were washed three times with TBST buffer and visualized via enzyme-linked chemiluminescence using an enhanced chemiluminescence kit (Applygen Technologies Inc., Beijing, China). The results of western blotting were semi-quantitatively analyzed by Image J software version 1.62 (National Institutes of Health, Bethesda, MD, USA). The protein levels were quantified using ImageJ software version 1.62 and the intensity values of the lanes were obtained to calculate the ratio between various cell types. Protein levels were normalized to $\beta$-actin.

Cell proliferation assay. Cell-Counting Kit- 8 colorimetric assay (Dojindo Molecular Technologies, Inc., Kumamoto, Japan) was used to evaluate cell proliferation and viability, according to a previous protocol (17). The experiments were repeated three times in quadruplicate wells in order to ensure the reproducibility of the results.

Wound healing assay. Cells were plated at a density of $2 \times 10^{5}$ cells/well in 6 -well plates and grown to $100 \%$ confluence. 
Table I. Clinical characteristics of patients with extrahepatic bile duct carcinoma.

\begin{tabular}{|c|c|}
\hline Characteristics & $\mathrm{N}(\%)$ \\
\hline Number of patients & 54 \\
\hline Mean age (range), years & $61.1(5-86)$ \\
\hline Gender, male/female & $29 / 25$ \\
\hline \multicolumn{2}{|l|}{ Pathological stage } \\
\hline I & $12(22.2)$ \\
\hline II & $18(33.3)$ \\
\hline III & $19(35.2)$ \\
\hline IV & $5(9.3)$ \\
\hline \multicolumn{2}{|l|}{ T stage } \\
\hline 1 & $13(24.1)$ \\
\hline 2 & $26(48.1)$ \\
\hline 3 & $13(24.1)$ \\
\hline 4 & $2(3.7)$ \\
\hline \multicolumn{2}{|l|}{$\mathrm{N}$ stage } \\
\hline N0 & $41(75.9)$ \\
\hline N1 & $11(20.4)$ \\
\hline N2 & $2(3.7)$ \\
\hline \multicolumn{2}{|l|}{ M stage } \\
\hline M0 & $0(0.0)$ \\
\hline M1 & $0(0.0)$ \\
\hline \multicolumn{2}{|l|}{ Differentiation degree } \\
\hline Well & $20(37.0)$ \\
\hline Moderate & $16(29.7)$ \\
\hline Poor & $18(33.3)$ \\
\hline
\end{tabular}

T, tumor; $\mathrm{N}$, node; $\mathrm{M}$, metastasis.

The cell monolayer was scratched with a $200-\mu 1$ pipette tip to create a wound gap, which was immediately imaged using an Olympus IX71 microscope (Olympus Corporation, Tokyo, Japan) at $0 \mathrm{~h}$. Migration was then observed every $12 \mathrm{~h}$, and the closing wound was imaged at each stage. Image-Pro Plus analysis software version 6.0.0.260 (Media Cybernetics, Inc., Rockville, MD, USA) was used to calculate the relative migration rate (\%) as follows: [(The relative distance recorded at $0 \mathrm{~h}$-the relative distance recorded at 12 and $24 \mathrm{~h}$ ) / the relative distance recorded at $0 \mathrm{~h}] \mathrm{x} 100$.

Statistical analysis. All experiments were repeated $\geq 3$ times. SPSS version 11.5 software (SPSS, Inc., Chicago, IL, USA) was used to analyze the results. All data were presented as the mean \pm standard deviation. Cell proliferation was evaluated by repeated-measures $t$-tests. Cell migration and comparison of EBP50 expression levels between extrahepatic bile duct carcinoma and normal extrahepatic bile duct epithelium was evaluated using independent sample $t$-tests. One-way analysis of variance was used to evaluate the correlation between EBP50 expression levels and clinical stages/differentiation degree. $\mathrm{P}<0.05$ was considered to indicate a statistically significant difference.
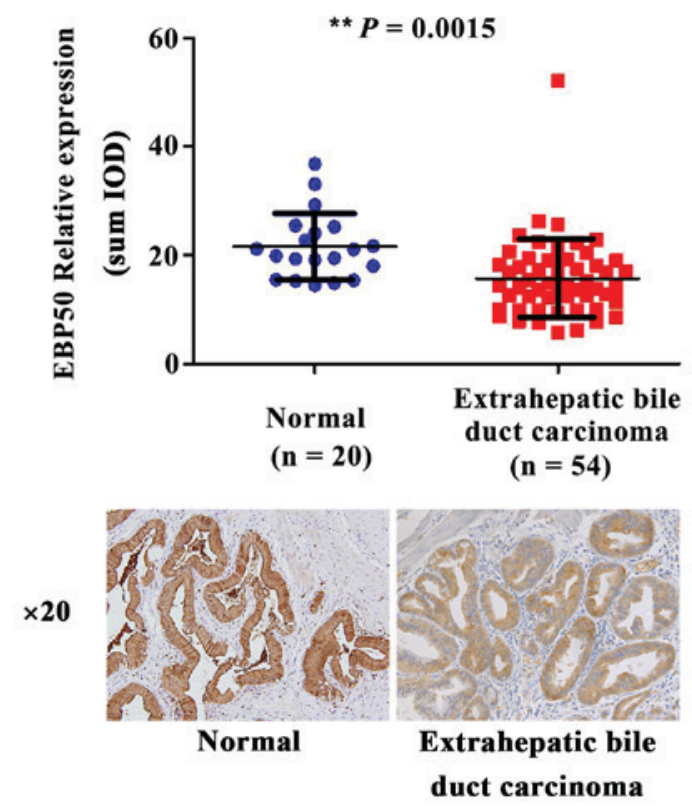

Figure 1. The expression levels of EBP50 were downregulated in extrahepatic bile duct carcinoma, as compared with those in normal extrahepatic bile duct epithelia from the gallbladder of patients with cholecystitis. Normal and extrahepatic bile duct carcinoma tissues underwent an IHC reaction with anti-EBP50 antibodies. The results are presented as representative IHC images. EBP50, ezrin-radixin-moesin-binding phosphoprotein-50; IHC, immunohistochemistry; sum IOD, sum of integrated optical density. ${ }^{* *} \mathrm{P}=0.0015$, normal vs. extrahepatic bile duct carcinoma.

\section{Results}

EBP50 expression levels are downregulated in extrahepatic bile duct carcinoma tissue. EBP50 has previously been observed to be involved in the development of multiple types of tumors (18). However, its effect on extrahepatic bile duct carcinoma tissues remains unclear. To elucidate the effect of EBP50 in extrahepatic bile duct carcinoma progression, EBP50 expression levels in the extrahepatic bile duct carcinoma tissues were investigated. IHC results demonstrated that EBP50 expression in the extrahepatic bile duct carcinoma tissues $(\mathrm{n}=54)$ was significantly downregulated $(\mathrm{P}=0.0015$; Fig. 1), as compared with normal extrahepatic bile duct epithelia from the gallbladder of patients with cholecystitis $(n=20)$. This suggests that EBP50 may be a tumor-associated protein in extrahepatic bile duct carcinoma, and that its expression levels are potentially associated with the occurrence and development of extrahepatic bile duct carcinoma.

EBP50 expression levels of are correlated with the pathological stage of extrahepatic bile duct carcinoma. To further evaluate the association between EBP50 expression levels and the pathological stage of extrahepatic bile duct carcinoma, the EBP50 expression profile was analyzed in tissues samples from various stages of extrahepatic bile duct carcinoma. The expression levels of EBP50 gradually decreased in extrahepatic bile duct carcinoma, corresponding with an increase in pathological stage (stages I/II-III/IV). This increase in 
pathological stage was positively associated with a decrease in EBP50 expression levels ( $\mathrm{P}=0.0016$; Fig. 2A). To elucidate whether EBP50 expression levels are correlated with $\mathrm{T}$ or $\mathrm{N}$ staging, the correlation between EBP50 expression levels in extrahepatic bile duct carcinoma and individual $\mathrm{T}$ and $\mathrm{N}$ stages was analyzed. As presented in Fig. 2B, compared with that at T1 stage, the expression levels of EBP50 were decreased at the $\mathrm{T} 2$ and $\mathrm{T} 3 / 4$ stage $(\mathrm{P}=0.0136)$. No significant differences were observed between the expression levels of EBP50 and the $\mathrm{N}$ stage of the tumor $(\mathrm{P}=0.1069)$. These results indicate that the expression levels of EBP50 may be correlated with the pathological stage of extrahepatic bile duct carcinoma, particularly with regard to overall staging and $\mathrm{T}$ staging, thus suggesting a positive correlation between low expression levels of EBP50 and malignant phenotype of extrahepatic bile duct carcinoma.

EBP50 expression levels are correlated with the differentiation degree of extrahepatic bile duct carcinoma. The differentiation degree of tumors is closely associated with prognosis (19). Therefore, the association between the tumor differentiation degree and the expression levels of EBP50 was further investigated. As presented in Fig. 3, the expression levels of EBP50 were highest in normal bile duct epithelia from the gallbladder of patients with cholecystitis, and the expression levels were gradually reduced along with a decrease in histological differentiation degree $(\mathrm{P}=0.0003)$. This result demonstrated the positive correlation between low expression levels of EBP50 and the malignant phenotype of extrahepatic bile duct carcinoma.

Generation of EBP50 knockdown cells. Clinicopathological data exhibited a positive correlation between low expression levels of EBP50 and the malignant phenotype of extrahepatic bile duct carcinoma, suggesting that EBP50 expression levels may regulate the development and progression of extrahepatic bile duct carcinoma. To elucidate whether low expression levels of EBP50 are associated with the development of extrahepatic bile duct carcinoma, EBP50 expression was knocked down in the QBC939 extrahepatic bile duct carcinoma cell line following transfection with siRNA duplexes directed against EBP50. QBC939 cells were also transfected with scrambled RNAi to establish control cells. EBP50 knockdown was identified by western blotting and is presented in Figs. 4A and 5B. The protein levels were quantified by Image J software version 1.48 and the intensity values of the lanes were obtained to provide the ratio between various cells. The QBC939 siEBP50 group expressed low levels of EBP50 protein, which comprised 1/3 and 1/5 of the protein level of EBP50 in the siRNA-NC group, respectively. These results suggest that EBP50 expression was significantly inhibited $(\mathrm{P}=0.012)$.

EBP50 knockdown promotes the proliferation of QBC939 cells. The effect of EBP50 knockdown on the proliferation of QBC939 cells was initially detected by determining the number of viable cells at various time points. The results demonstrated that the knockdown of EBP50 significantly promoted cell proliferation, as compared with the vector control cells $(\mathrm{P}=0.04$; Fig. 4B). These results suggest that decreased EBP50
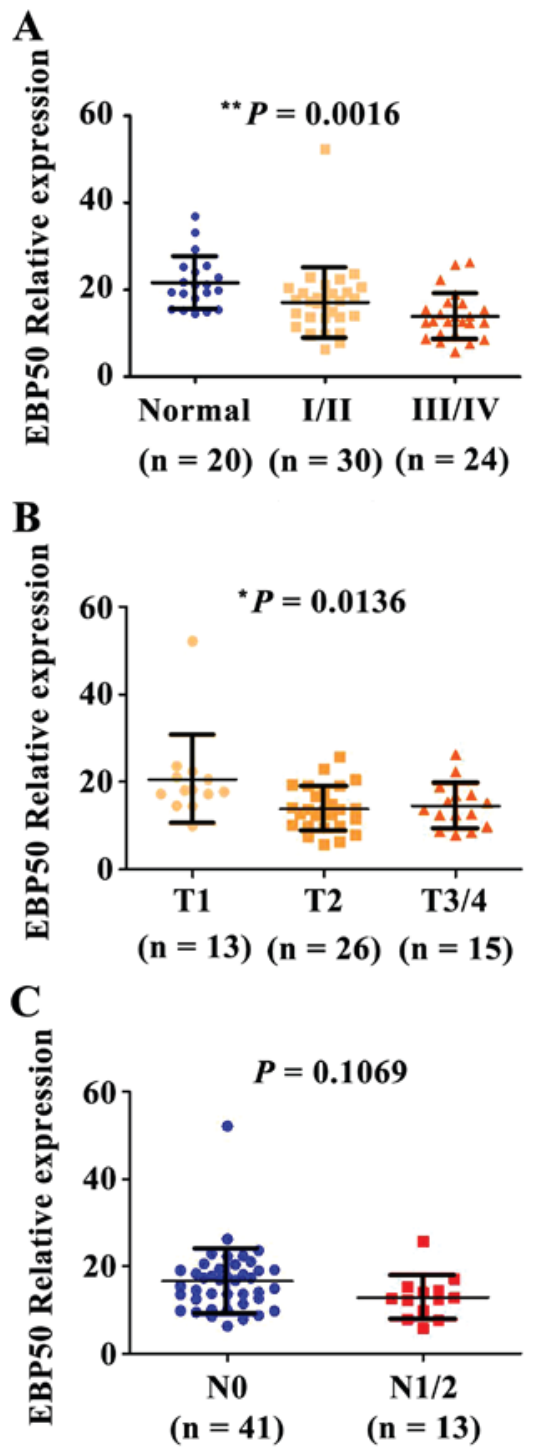

Figure 2. The expression levels of EBP50 decreased with the increase in pathological stage or T classification of extrahepatic bile duct carcinoma. The normal and extrahepatic bile duct carcinoma tissues underwent an IHC reaction with anti-EBP50 antibodies. The variation in EBP50 expression levels among (A) pathological stage, (B) T classification and (C) N classification was examined using analysis of variance, $\mathrm{P}=0.1069$ between $\mathrm{N} 0$ and N1/2. ${ }^{* *} \mathrm{P}=0.0016$ between normal, stage I/II and stage III/IV, ${ }^{*} \mathrm{P}=0.0136$ among T1, T2 and T3/4 EBP50, ezrin-radixin-moesin-binding phosphoprotein-50; IHC, immunohistochemistry; T, tumor; $\mathrm{N}$, node.

expression levels may be one of the mechanisms underlying the occurrence of CC.

EBP50 knockdown promotes the migration of QBC939 cells. Cell migration and invasion are also implicated in the development of tumors $(19,20)$. Therefore, the potential of EBP50 knockdown to promote cell migration was investigated. During a wound-healing assay, no significant change in wound width was observed in the control group, whereas the wound width was significantly reduced in the EBP50 knockdown group $(\mathrm{P}=0.02) 12$ and $24 \mathrm{~h}$ following wounding (Fig. 5B). These results suggest that EBP50 knockdown was able to promote the migration of QBC939 cells, and additionally demonstrated that decreased expression levels of EBP50 may be a potential factor in the progression of CC. 


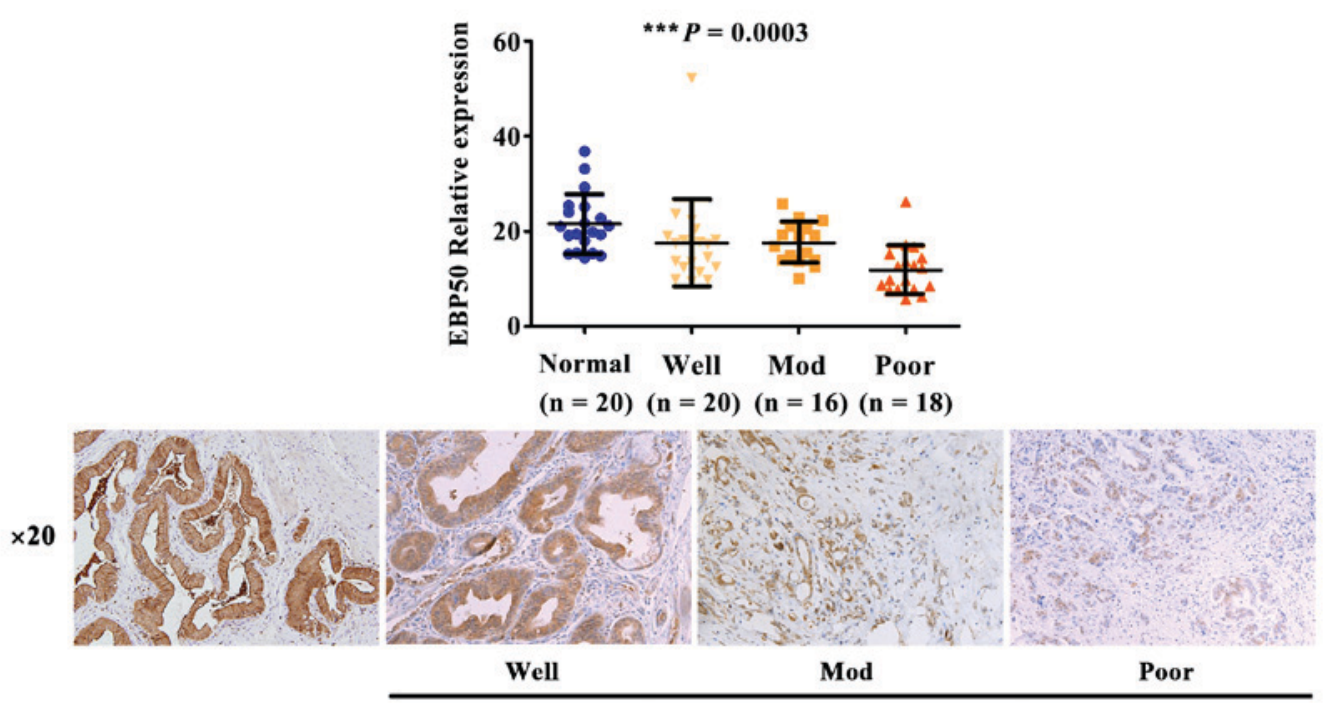

Normal

Extrahepatic bile duct carcinoma

Figure 3. The expression levels of EBP50 gradually decreased, corresponding with a reduction in the differentiation degree, in extrahepatic bile duct carcinoma. Normal and extrahepatic bile duct carcinoma tissues underwent immunohistochemistry reactions with anti-EBP50 antibodies. The difference in EBP50 expression levels among normal and tumor tissues with varying differentiation degrees was evaluated using analysis of variance. EBP50, ezrin-radixin-moesin-binding phosphoprotein-50; Well, well differentiated; Mod, moderately differentiated; Poor, poorly differentiated. ${ }^{* * *} \mathrm{P}=0.0003$ between normal, well, mod and poor differentiation degrees.

A
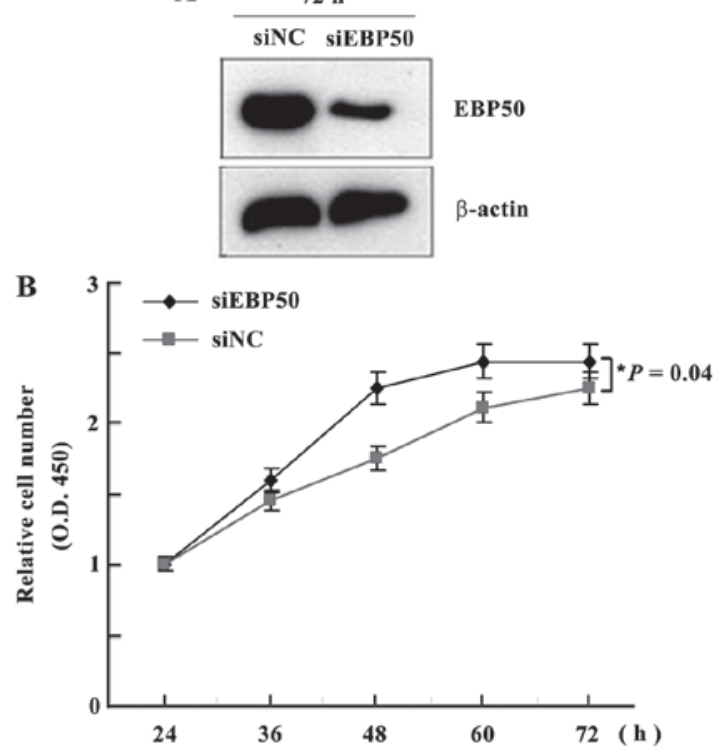

Figure 4. EBP50 knockdown promoted QBC939 cell proliferation. Cell counting kit-8 assay was performed to analyze the proliferation of control cells and cells transfected with siRNA-EBP50 at various time points. (A) EBP50 expression levels were significantly knocked down. (B) QBC939 cell proliferation was significantly promoted. ${ }^{*} \mathrm{P}=0.04$ between siNC and siEBP50. EBP50, ezrin-radixin-moesin-binding phosphoprotein-50; siRNA, small interfering RNA; NC, negative control; OD, optical density.

\section{Discussion}

In the present study, the expression levels of EBP50 in CC tissues and normal bile duct epithelia from the gallbladder of patients with cholecystitis were examined, in addition to the phenotypic changes that resulted from EBP50 knockdown in
A
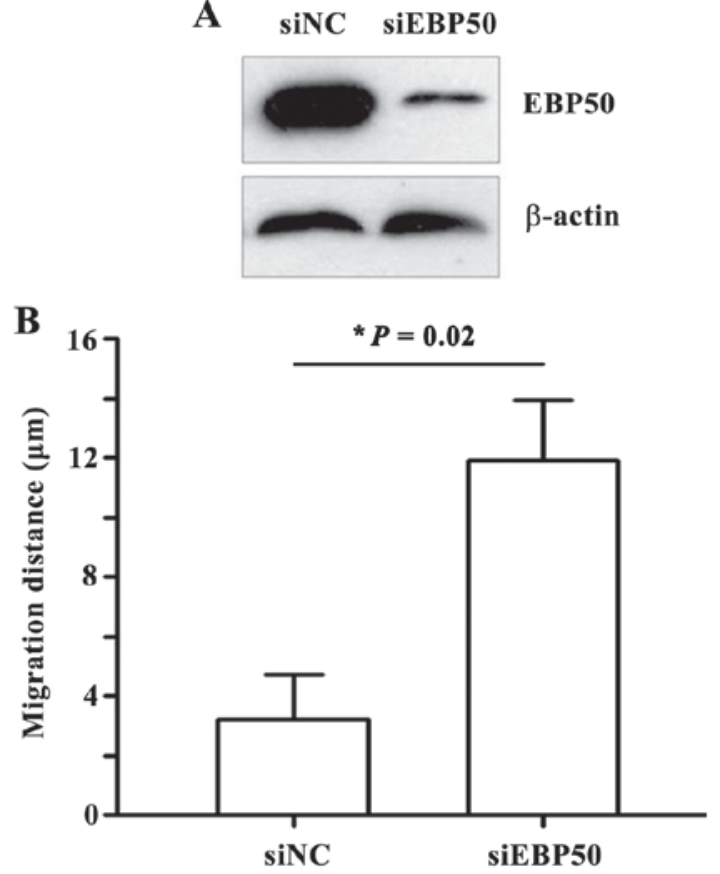

Figure 5. EBP50 knockdown enhanced the migratory ability of QBC939 cells. A wound-healing assay was performed using control cells and cells transfected with siRNA-EBP50. siRNA-EBP50 QBC939 cells migrated significantly further than siRNA-NC QBC939 cells. ${ }^{~} \mathrm{P}=0.02$ between siNC and siEBP50. EBP50, ezrin-radixin-moesin-binding phosphoprotein-50; siRNA, small interfering RNA; NC, negative control.

QBC939 cells. The results demonstrated that the expression of EBP50 was downregulated in extrahepatic bile duct carcinoma, as compared with that in normal bile duct epithelia from the gallbladder. In addition, low EBP50 expression levels were positively correlated with poor differentiation and advanced 
clinical stages in extrahepatic bile duct carcinoma. EBP50 knockdown significantly promoted the proliferative and migratory abilities of QBC939 cells. Therefore, EBP50 may potentially exert a tumor-suppressing function in extrahepatic bile duct carcinoma.

Abnormalities in EBP50 expression have been demonstrated to be associated with the occurrence, development and metastasis of certain types of cancer (18). Using IHC, the present study demonstrated that EBP50 protein expression levels were dysregulated in extrahepatic bile duct cancer tissue samples. Data from The Cancer Genome Atlas database (https://cancergenome.nih.gov/) also indicated that EBP50 messenger (m)RNA expression is dysregulated in extrahepatic bile duct cancer tissue samples, and that EBP50 mRNA expression levels gradually decreased with the increase in $\mathrm{T}$ or $\mathrm{N}$ stage (data not shown). However, in the present study, no significant differences were observed between EBP50 mRNA expression levels and changes in TNM staging. This may be due to the small number of CC cases or the various sources of cases, and requires further investigation.

The high expression levels of EBP50 observed in the bile duct epithelia from the gallbladder were concordant with the results of a previous study by Fouassier et al (21). In that study, the expression levels of EBP50 in the gallbladder were 40-fold higher, compared with those observed in intrahepatic bile ducts. Furthermore, the high expression levels of EBP50 in the extrahepatic bile ducts presented a marked contrast with the low expression levels of EBP50 in the intrahepatic bile ducts (21). Yonglitthipagon et al (22) demonstrated that EBP50 is expressed at low levels in liver tissue and at high levels in intrahepatic bile duct cancer tissues. Due to the variation in the control samples, the present study produced contrasting results and drew an alternative conclusion. In the study by Yonglitthipagon et al (22), CC was associated with liver fluke infections. The variation in the genetic mutation landscape and gene expression profiles between cancer in endemic areas and non-endemic areas of liver fluke infection may also be the reason for the alternate role of EBP50 in $\mathrm{CC}$, and requires further study.

In the present study, EBP50 expression in the QBC939 human $\mathrm{CC}$ cell line was effectively knocked down by transfection with EBP50 siRNA. EBP50 knockdown promoted the proliferative and migratory abilities of QBC939 cells. Cell proliferation and migration are associated with tumor development, and are the primary causes of mortality in patients with CC (23). Therefore, a possible explanation for the positive correlation between reduced EBP50 expression levels and unfavorable clinicopathological features in extrahepatic bile duct carcinoma is provided.

As a scaffold protein, EBP50 has diverse functions through binding to various proteins, including postsynaptic density protein 95, Drosophila disc large tumor suppressor and zonula occludens-1 protein domain proteins (24). EBP50 is considered to indirectly or directly affect cancer behaviors through the modification of signal transduction $(12,14,15,25-27)$, and inhibits EGF-induced breast cancer cell proliferation by blocking the phosphorylation of EGFR (25). This inhibitory role of EBP50 in the EGF/EGFR signaling pathway has also been demonstrated in biliary cancer cells, where the loss of EBP50 promotes EGFR activity and induces the appearance of EMT phenotypic features (15). Regarding the underlying molecular mechanism by which EBP50 may inhibit the proliferation and migration of CC cells, it is possible that EBP50 interacts with signaling molecules that are important in migration and proliferation, and may regulate the functions of its binding partners. EBP50 may potentially interact with EGFR in order to inhibit the proliferation and migration of biliary cancer cells. In addition, EBP50 has been demonstrated to be an actin cytoskeleton and cell junction-associated protein $(28,29)$. Abnormal expression and organization of the actin cytoskeleton and cell junctions are associated with tumor metastasis (30). Therefore, the potential metastasis-suppressing ability of EBP50 may result from its regulation of the actin cytoskeleton and cell junction proteins; however, these hypotheses require further investigation.

In conclusion, the present study demonstrated that the expression levels of EBP50 were downregulated in CC compared with those in normal bile duct tissue, and that low expression levels of EBP50 were correlated with poor-differentiated histological classes and advanced clinical stages of CC. In addition, EBP50 is able to regulate the malignant behaviors (proliferation and migration abilities) of QBC939 CC cells, concordant with the hypothesis that EBP50 functions as a tumor-suppressing protein in CC. Therefore, EBP50 may be a potential prognostic factor and therapeutic target in the treatment of CC. However, the molecular mechanisms underlying the effect of EBP50 on CC cell proliferation and migration have yet to be elucidated, and additional studies are required.

\section{Acknowledgements}

The present study was supported by funding from the National Natural Science Foundation of China (grant no. 81471652), the Postdoctoral Science Foundation of China (grant no. 2013M541208), the Natural Science Foundation of Shanxi province (grant no. 2014011037-3) and the Tumor Invasion and Metastasis Research Foundation of Beijing Key Laboratory (grant no. 2015ZLQX04).

\section{References}

1. Uppal DS and Wang AY: Advances in endoscopic retrograde cholangiopancreatography for the treatment of cholangiocarcinoma. World J Gastrointest Endosc 7: 675-687, 2015.

2. Hammill CW and Wong LL: Intrahepatic cholangiocarcinoma: A malignancy of increasing importance. J Am Coll Surg 207: 594-603, 2008.

3. Aljiffry M, Abdulelah A, Walsh M, Peltekian K, Alwayn I and Molinari M: Evidence-based approach to cholangiocarcinoma: A systematic review of the current literature. J Am Coll Surg 208: 134-147, 2009.

4. Nakeeb A, Tran KQ, Black MJ, Erickson BA, Ritch PS Quebbeman EJ, Wilson SD, Demeure MJ, Rilling WS, Dua KS and Pitt HA: Improved survival in resected biliary malignancies. Surgery 132: 555-564, 2002.

5. Morimoto Y, Tanaka Y, Ito T, Nakahara M, Nakaba H, Nishida T, Fujikawa M, Ito T, Yamamoto S and Kitagawa T: Long-term survival and prognostic factors in the surgical treatment for intrahepatic cholangiocarcinoma. J Hepatobiliary Pancreat Surg 10: 432-440, 2003.

6. Gerhards MF, van Gulik TM, Bosma A, ten Hoopen-Neumann H, Verbeek PC, Gonzalez Gonzalez D, de Wit LT and Gouma DJ: Long-term survival after resection of proximal bile duct carcinoma (Klatskin tumors). World J Surg 23: 91-96, 1999.

7. Shaib Y and El-Serag HB: The epidemiology of cholangiocarcinoma. Semin Liver Dis 24: 115-125, 2004. 
8. Lazaridis KN and Gores GJ: Cholangiocarcinoma. Gastroenterology 128: 1655-1667, 2005.

9. Olnes MJ and Erlich R: A review and update on cholangiocarcinoma. Oncology 66: 167-179, 2004.

10. Khan SA, Davidson BR, Goldin RD, Pereira SP, Rosenberg WM Taylor-Robinson SD, Thillainayagam AV, Thomas HC, Thursz MR and Wasan H; British Society of Gastroenterology: Guidelines for the diagnosis and treatment of cholangiocarcinoma: Consensus document. Gut 51 (Suppl 6): VI1-VI9, 2002.

11. Brandi G, Venturi M, Pantaleo MA and Ercolani G; GICO Cholangiocarcinoma: Current opinion on clinical practice diagnostic and therapeutic algorithms: A review of the literature and a long-standing experience of a referral center. Dig Liver Dis 48 : 231-241, 2016.

12. Shibata T, Chuma M, Kokubu A, Sakamoto M and Hirohashi S: EBP50, a beta-catenin-associating protein, enhances Wnt signaling and is over-expressed in hepatocellular carcinoma. Hepatology 38: 178-186, 2003

13. Saponaro C, Malfettone A, Dell'Endice TS, Brunetti AE, Achimas-Cadariu P, Paradiso A and Mangia A: The prognostic value of the $\mathrm{Na}^{+} / \mathrm{H}^{+}$exchanger regulatory factor 1 (NHERF1) protein in cancer. Cancer Biomark 14: 177-184, 2014.

14. Peng XL, Ji MY, Yang ZR, Song J and Dong WG: Tumor suppressor function of ezrin-radixin-moesin-binding phosphoprotein-50 through $\beta$-catenin/E-cadherin pathway in human hepatocellular cancer. World J Gastroenterol 19: 1306-1313, 2013.

15. Clapéron A, Guedj N, Mergey M, Vignjevic D, Desbois-Mouthon C, Boissan M, Saubaméa B, Paradis V, Housset C and Fouassier L: Loss of EBP50 stimulates EGFR activity to induce EMT phenotypic features in biliary cancer cells. Oncogene 31: 1376-1388, 2012

16. Edge SB, Byrd DR, Compton CC, et al (eds.): AJCC cancer staging manual. 7. Carcinoma of the eyelid. Springer, New York, USA, pp 523-530, 2009.

17. Zheng JF, Sun LC, Liu H, Huang Y, Li Y and He J: EBP50 exerts tumor suppressor activity by promoting cell apoptosis and retarding extracellular signal-regulated kinase activity. Amino Acids 38: 1261-1268, 2010.

18. Georgescu MM, Morales FC, Molina JR and Hayashi Y: Roles of NHERF1/EBP50 in cancer. Curr Mol Med 8: 459-468, 2008.

19. Barresi V, Reggiani Bonetti L, Ieni A, Domati F and Tuccari G: Prognostic significance of grading based on the counting of poorly differentiated clusters in colorectal mucinous adenocarcinoma. Hum Pathol 46: 1722-1729, 2015.

20. Atanasov G, Hau HM, Dietel C, Benzing C, Krenzien F, Brandl A, Wiltberger G, Matia I, Prager I, Schierle K, et al: Prognostic significance of macrophage invasion in hilar cholangiocarcinoma. BMC Cancer 15: 790, 2015.
21. Fouassier L, Rosenberg P, Mergey M, Saubaméa B, Clapéron A, Kinnman N, Chignard N, Jacobsson-Ekman G, Strandvik B, Rey $\mathrm{C}$, et al: Ezrin-radixin-moesin-binding phosphoprotein (EBP50), an estrogen-inducible scaffold protein, contributes to biliary epithelial cell proliferation. Am J Pathol 174: 869-880, 2009.

22. Yonglitthipagon P, Pairojkul C, Chamgramol Y, Loukas A, Mulvenna J, Bethony J, Bhudhisawasdi V and Sripa B: Prognostic significance of peroxiredoxin 1 and ezrin-radixin-moesin-binding phosphoprotein 50 in cholangiocarcinoma. Hum Pathol 43: 1719-1730, 2012.

23. Kreimann EL, Morales FC, de Orbeta-Cruz J, Takahashi Y, Adams H, Liu TJ, McCrea PD and Georgescu MM: Cortical stabilization of beta-catenin contributes to NHERF1/EBP50 tumor suppressor function. Oncogene 26: 5290-5299, 2007.

24. Bian W, Sun L, Yang L, Li JF, Hu J, Zheng S, Guo R, Feng D, Ma Q, Shi X, et al: Stabilization of the angiotensin-(1-7) receptor Mas through interaction with PSD95. Biochem J 453: 345-356, 2013.

25. Yao W, Feng D, Bian W, Yang L, Li Y, Yang Z, Xiong Y, Zheng J, Zhai R and He J: EBP50 inhibits EGF-induced breast cancer cell proliferation by blocking EGFR phosphorylation. Amino Acids 43: 2027-2035, 2012

26. Cheng S, Li Y, Yang Y, Feng D, Yang L, Ma Q, Zheng S, Meng R, Wang S, Wang S, et al: Breast cancer-derived K172N, D301V mutations abolish $\mathrm{Na}+\mathrm{H}+$ exchanger regulatory factor 1 inhibition of platelet-derived growth factor receptor signaling. FEBS Lett 587: 3289-3295, 2013.

27. Yang L, Wang Y, Chen P, Hu J, Xiong Y, Feng D, Liu H, Zhang H, Yang $\mathrm{H}$ and $\mathrm{He} \mathrm{J}: \mathrm{Na}(+) / \mathrm{H}(+)$ exchanger regulatory factor 1 (NHERF1) is required for the estradiol-dependent increase of phosphatase and tensin homolog (PTEN) protein expression. Endocrinology 152: 4537-4549, 2011.

28. Hałon A and Donizy P: The role of the ERM protein family in maintaining cellular polarity, adhesion and regulation of cell motility. Postepy Hig Med Dosw (Online) 66: 158-164, 2012 (In Polish).

29. Sun L, Zheng J, Wang Q, Song R, Liu H, Meng R, Tao T, Si Y, Jiang $\mathrm{W}$ and He J: NHERF1 regulates actin cytoskeleton organization through modulation of alpha-actinin- 4 stability. FASEB J 30: 578-589, 2016.

30. Jawhari AU, Buda A, Jenkins M, Shehzad K, Sarraf C, Noda M, Farthing MJ, Pignatelli M and Adams JC: Fascin, an actin-bundling protein, modulates colonic epithelial cell invasiveness and differentiation in vitro. Am J Pathol 162: 69-80, 2003. 